Acta Crystallographica Section D

\section{Biological \\ Crystallography}

ISSN 1399-0047

\section{Jong Hwan Yoon, ${ }^{a}$ Young-jin Chob and Hyun Ho Park ${ }^{\mathrm{a} *}$}

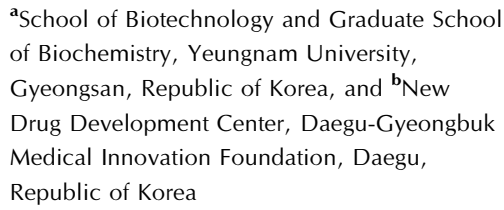

Correspondence e-mail: hyunho@ynu.ac.kr

\title{
Structure of the TRAF4 TRAF domain with a coiled-coil domain and its implications for the TRAF4 signalling pathway
}

The TNF receptor-associated factor (TRAF) proteins are structurally similar scaffold proteins that mediate between members of the TNF receptor (TNFR) family and downstream effector molecules such as kinases in the immune signalling pathway. Seven TRAFs have been identified, including TRAF4, which is a unique member that participates in many ontogenic processes, including nerve-system development. TRAFs commonly contain the TRAF domain, which mediates interaction with target receptors and effectors. As a first step towards elucidating the molecular mechanisms of the TRAF4-mediated signalling pathway, the first crystal structure of the human TRAF4 TRAF domain with a coiled-coil domain is reported at $2.3 \AA$ resolution.

\section{Introduction}

The tumour necrosis factor (TNF) receptor-associated factor (TRAF) proteins are important cellular signalling molecules in the tumour necrosis factor receptor (TNFR) and Toll-like receptor (TLR) family signalling pathways that play a critical role in the regulation of inflammation, apoptosis and antiviral response (Inoue et al., 2000; Chung et al., 2002; Bradley \& Pober, 2001). TRAFs are also involved in the proper function of many immune cells, including CD40-expressing antigenpresenting cells such as B lymphocytes, macrophages and dendritic cells (Rothe et al., 1995; Bishop, 2004). TRAFs function as scaffold proteins that link receptors to downstream signalling molecules, which are primarily protein kinases and include interleukin-1 receptor-associated kinases (IRAKs), receptor-interacting protein 1 (RIP1), RIP2, transforming growth factor $\beta$-activated kinase 1 (TAK1), mitogen-activated protein kinase kinase kinase 1 (MEKK1) and apoptosis signalregulating kinase 1 (ASK1) (Arch et al., 1998; Hsu et al., 1996; Song et al., 1997; Hoeflich et al., 1999). Several ubiquitin ligases are also recruited to membrane receptors via direct interaction with TRAFs (Song et al., 1996). Antagonistic functions of TRAFs in the TNFR and TLR signalling pathways have also been reported by showing that several TRAFs were not able to recruit any downstream effectors (Ye, Mehlen et al., 1999; Zapata et al., 2001). Owing to their involvement in many human diseases, including cancer, autoimmunity and inflammatory diseases, TRAFs have been suggested to be suitable targets for therapeutic intervention (Zapata et al., 2007).

Seven TRAF proteins have been identified in mammals (TRAF1-TRAF7; Chung et al., 2002; Xie, 2013), all of which
Received 17 June 2013

Accepted 19 August 2013

PDB Reference: human TRAF4 TRAF domain, 4k8u 
contain the TRAF domain with the exception of TRAF7, sequence analysis of which indicated that it is unlikely to contain a TRAF domain. The TRAF domain is an eightstranded antiparallel $\beta$-sandwich structure composed of around 180 amino acids (Rothe et al., 1994). The TRAF domain mediates self-association and upstream interactions with receptors and other signalling molecules. Despite the structural similarity of the TRAF domain, each TRAF protein exhibits specific biological functions, possessing specificity in its interactions with upstream receptors and downstream effector molecules. For instance, TRAF6 is known as a key adaptor molecule in the receptor activator of the NF- $\kappa \mathrm{B}$ (RANK)/RANK ligand (RANKL) signalling pathway, which is critical to regulation of bone modelling and lymph-node organogenesis (Darnay et al., 1999).

Even though TRAF4 is one of the canonical TRAF proteins, it contains unique domain boundaries and has unique functions in the cell (Kedinger \& Rio, 2007). Only TRAF4 contains the unique N-terminal RING-finger motif and a nuclear localization signal (NLS). In addition, the TRAF4 TRAF domain contains the shortest coiled-coil domain among the TRAFs (Xie, 2013). A structure-based sequence-alignment study showed that the receptor-interaction hot spot in TRAF3 is conserved in TRAF2 and TRAF5 but is not conserved in TRAF4 and TRAF6. This may indicate that TRAF4 can accommodate novel receptors or binding partners, leading to a novel signalling pathway (Ely \& $\mathrm{Li}, 2002$ ). Although the cellular functions of TRAF4 are not well understood, several studies, including a mouse knockout study, have indicated that it is involved in gross tracheal, neural tube and skeletal formation (Kedinger \& Rio, 2007; Shiels et al., 2000; Régnier et al., 2002). While most TRAF-knockout mice showed alteration of the immune system, TRAF4-deficient and TRAF6deficient mice exhibited nervous alterations. The most recent studies have also shown that TRAF4 specifically inhibits the activation of NF- $\kappa$ B by interacting with NOD-like receptors (Marinis et al., 2011) and is particularly involved in IL-17 signalling and Th17-mediated disease (Zepp et al., 2012).

Previously reported crystal structures of the TRAF domains of TRAF2 (Park et al., 1999), TRAF3 (Zhang et al., 2012), TRAF5 (Zhang et al., 2012) and TRAF6 (Ye et al., 2002) have shown that TRAF domains are composed of an antiparallel 7-8 $\beta$-sheet fold followed by a coiled-coil region. TRAF domains usually form mushroom-like trimeric structures in solution. Despite the emerging roles of TRAF4 in human disease states, limited structural information is available (Izban et al., 2000; Aston et al., 2004).

In this study, we report the first crystal structure of the TRAF4 TRAF domain with an extra coiled-coil domain comprising amino acids $290-470$ at $2.3 \AA$ resolution. Although the TRAF4 TRAF domain has the typical TRAF-domain fold, the high-resolution structure reveals both similarities and differences between this structure and those of other TRAF family members, which may be functionally relevant. Further structure and sequence analysis revealed that the conserved surface residues of the TRAF4 TRAF domain might be critical for interaction with signalling molecules.
Table 1

Crystallographic statistics.

Values in parentheses are for the highest resolution shell.

\begin{tabular}{ll}
\hline Data collection & \\
Space group & $P 2_{1} 2_{1} 2_{1}$ \\
Unit-cell parameters $(\AA)$ & $a=58.9, b=87.9, c=117.3$ \\
Resolution $(\AA)$ & $50-2.3$ \\
$R_{\text {merge }}(\%)$ & $8.0(56.9)$ \\
$\langle I / \sigma(I)\rangle$ & $43.0(4.2)$ \\
$\quad$ Completeness $(\%)$ & $98.9(98.0)$ \\
$\quad$ Multiplicity & $8.9(8.9)$ \\
Refinement & \\
$\quad$ Resolution $(\AA)$ & $42-2.3$ \\
$\quad$ No. of reflections used/completeness $(\%)$ & $27524 / 99.1$ \\
$R_{\text {work }} / R_{\text {free }}(\%)$ & $23.7 / 28.0$ \\
No. of atoms & \\
$\quad$ Protein & 4294 \\
$\quad$ Water and other small molecules & 46 \\
Average $B$ factors $\left(\AA^{2}\right)$ & \\
$\quad$ Protein & 61.0 \\
$\quad$ Water and other small molecules & 19.8 \\
R.m.s. deviations & \\
$\quad$ Bond lengths $(\AA)$ & 0.010 \\
$\quad$ Bond angles $\left({ }^{\circ}\right)$ & 1.451 \\
Ramachandran plot, residues in $(\%)$ & 85.5 \\
$\quad$ Most favoured regions & 14.5 \\
$\quad$ Additional allowed regions & \\
\hline
\end{tabular}

\section{Materials and methods}

\subsection{Protein expression and purification}

The expression and purification methods used in this study have been described in detail elsewhere (Yoon \& Park, 2013). In summary, the human TRAF4 TRAF domain with a coiledcoil domain comprising amino acids 290-470 was cloned into pET24a vector and expressed in Escherichia coli BL21 (DE3) cells by overnight induction at $293 \mathrm{~K}$. The protein contained a carboxy-terminal His tag and was purified by nickel-affinity and gel-filtration chromatography. A Superdex 200 10/30 gel-filtration column (GE Healthcare) pre-equilibrated with $20 \mathrm{~m} M$ Tris- $\mathrm{HCl} \mathrm{pH} 8.0,150 \mathrm{mM} \mathrm{NaCl}$ was used for gelfiltration chromatography. The protein, which eluted at around $15 \mathrm{ml}$ upon gel-filtration chromatography, was collected and concentrated to $9-10 \mathrm{mg} \mathrm{ml}^{-1}$ for crystallization.

\subsection{MALS}

The absolute molar mass of the TRAF4 TRAF domain with a coiled-coil domain was determined by multi-angle light scattering (MALS). The target protein was loaded onto a Superdex 200 HR 10/30 gel-filtration column (GE Healthcare) pre-equilibrated in a buffer consisting of $20 \mathrm{~m} M$ Tris- $\mathrm{HCl} \mathrm{pH}$ $8.0,150 \mathrm{~m} M \mathrm{NaCl}$. The ÄKTA chromatography system was coupled to a MALS detector (miniDAWN TREOS; Wyatt) and a refractive-index detector (Optilab DSP; Wyatt).

\subsection{Crystallization and data collection}

Crystallization was conducted at $293 \mathrm{~K}$ by the hanging-drop vapour-diffusion method using various screening kits. The final crystals used for the X-ray diffraction study were grown in plates by equilibrating a mixture consisting of $1 \mu \mathrm{l}$ protein

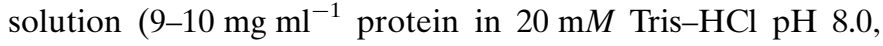


$150 \mathrm{~m} M \mathrm{NaCl})$ and $1 \mu \mathrm{l}$ reservoir solution ( $0.14 \mathrm{M}$ magnesium formate dehydrate, $13 \%$ polyethylene glycol 3350) against $0.4 \mathrm{ml}$ reservoir solution. A $2.3 \AA$ resolution native data set was collected using synchrotron radiation of wavelength $0.97950 \AA$ on the BL-4A beamline at Pohang Accelerator Laboratory (PAL), Republic of Korea. Data processing and scaling was carried out using $H K L-2000$ (Otwinowski \& Minor, 1997)

\subsection{Structure determination and analysis}

The structure was determined by the molecular-replacement phasing method using Phaser (McCoy, 2007). The previously solved structure of TRAF5 (PDB entry 4gjh; Zhang et al., 2012), which shares 33\% sequence identity with TRAF4, was used as a search model. Model building and refinement were performed in Coot (Emsley \& Cowtan, 2004) and REFMAC5 (Murshudov et al., 2011), respectively. Water molecules were

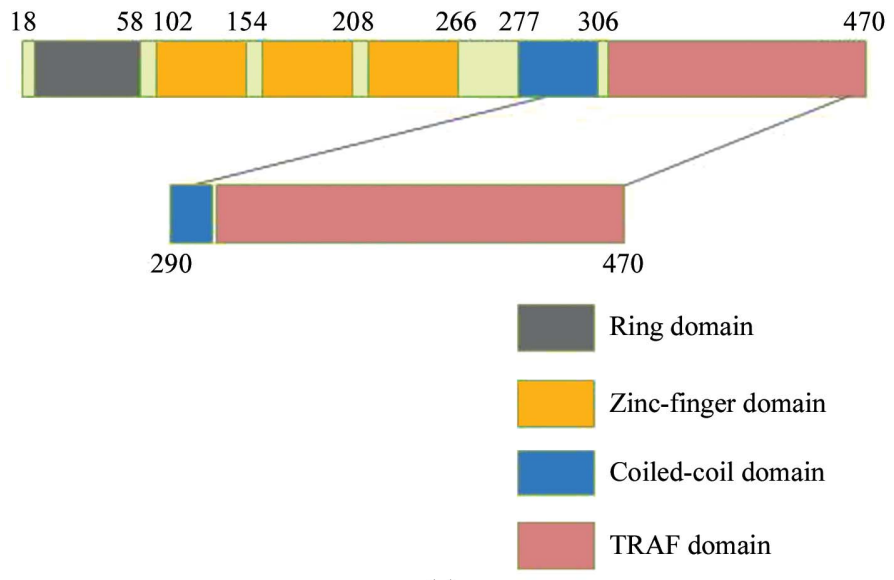

(a)

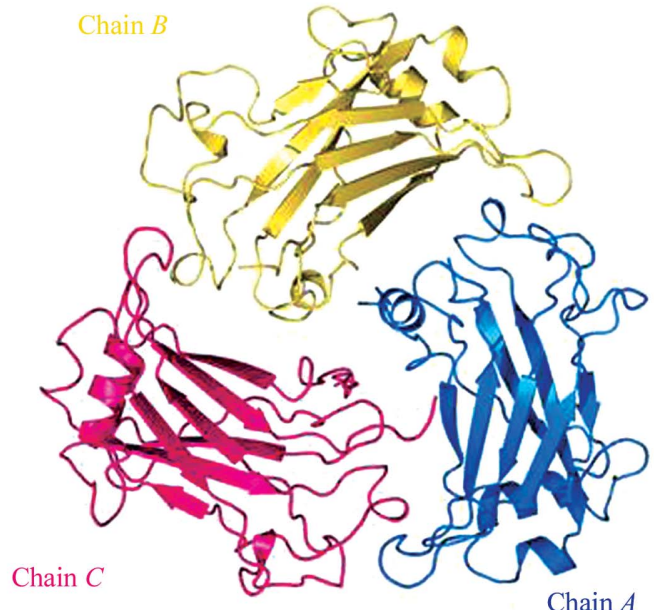

Figure 1

(c) added automatically using the $A R P / w A R P$ function in REFMAC5 and were then examined manually for reasonable hydrogen-bonding possibilities (Perrakis et al., 2001). The quality of the model was checked using PROCHECK and was found to be reasonable (Laskowski et al., 1993). A total of $85.5 \%$ of the residues were shown to be located in the most favourable region of the Ramachandran plot, while $14.5 \%$ were in allowed regions. The data-collection and refinement statistics are summarized in Table 1. Ribbon diagrams and molecular-surface representations were generated by $P y M O L$ (DeLano \& Lam, 2005).

\subsection{Sequence alignment}

The amino-acid sequences of TRAFs were analyzed using ClustalW (http://www.ebi.ac.kr/Tools/clustalw2/index.html).

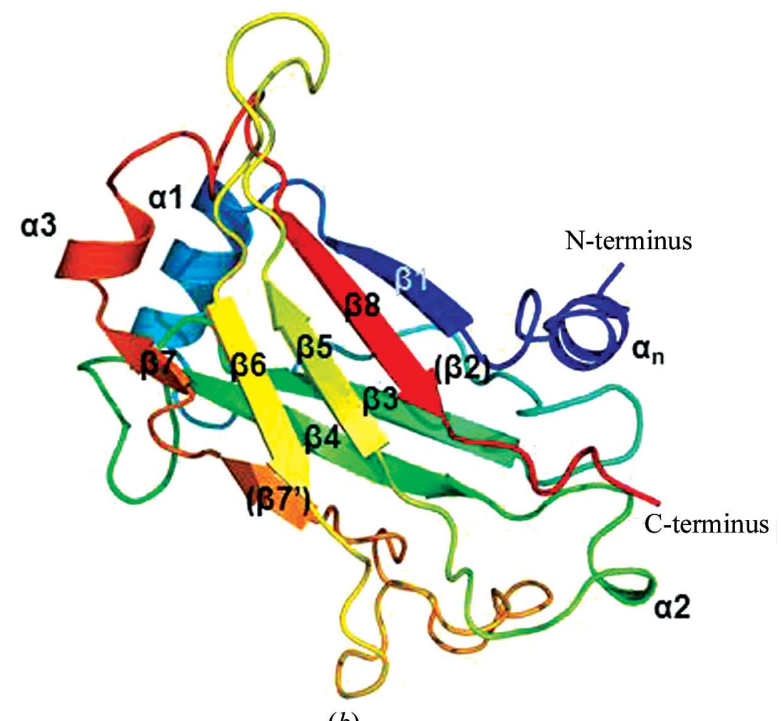

(b)

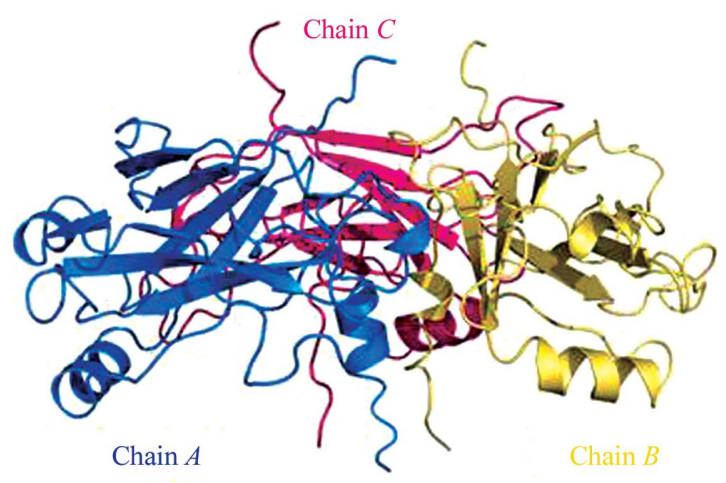

Chain $A$

Crystal structure of the TRAF4 TRAF domain with a coiled-coil domain. (a) Domain boundary of TRAF4. The TRAF4 construct containing residues 290-470 used in the current structural study is magnified. (b) Cartoon figure of the monomeric TRAF4 TRAF domain with a coiled-coil domain. The chain is coloured from blue to red from the N-terminus to the C-terminus. Secondary structures including helices and sheets are labelled. (c) Ribbon diagram of the trimeric TRAF4 TRAF domain with a coiled-coil domain. Chain $A$, chain $B$ and chain $C$ are shown separately in different colours. A top view and a side view are shown on the left and on the right, respectively. 


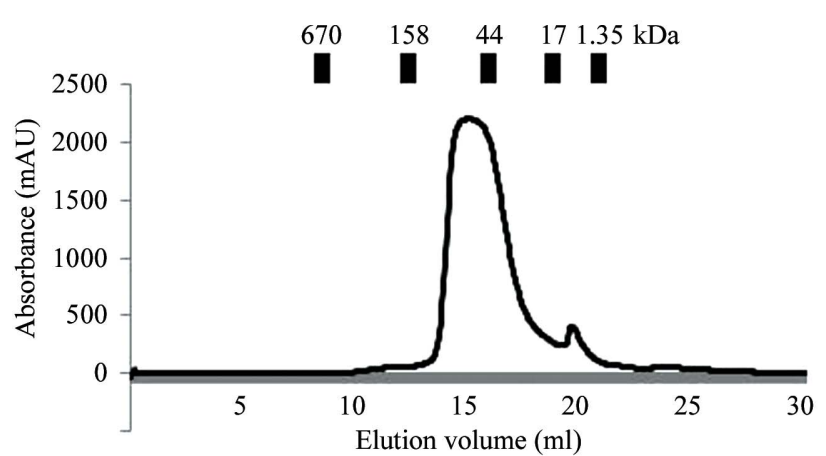

(a)

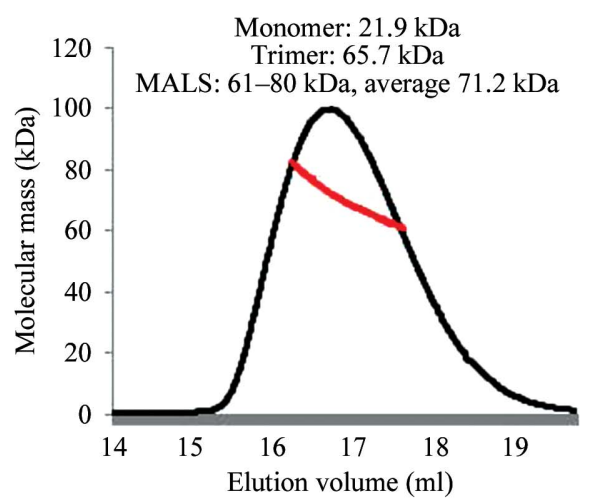

(b)

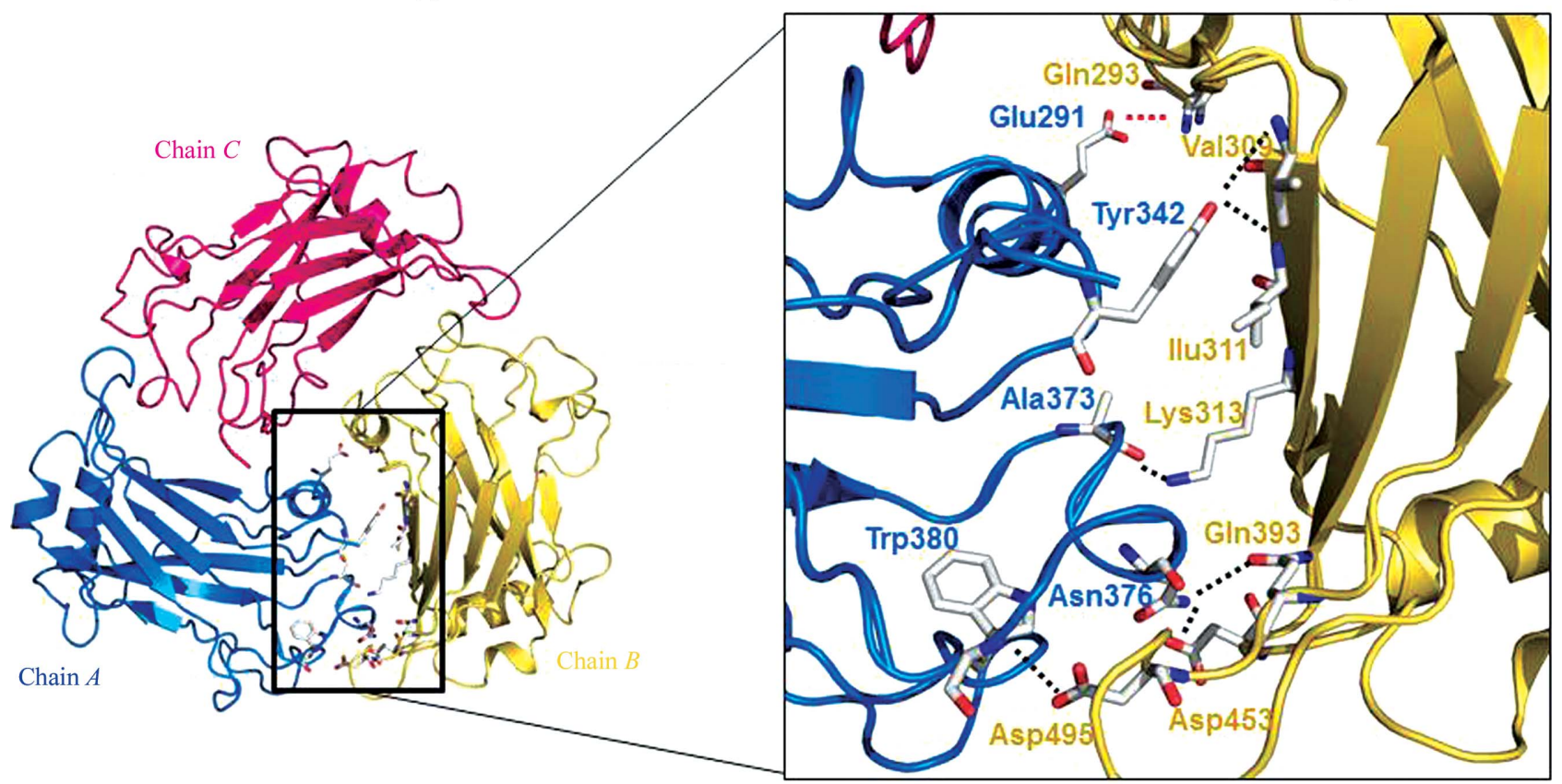

(c)

Figure 2

Stoichiometry and trimeric interface of the structure of the TRAF4 TRAF domain with a coiled-coil domain. (a) Gel-filtration chromatography profile. (b) Multi-angle light scattering (MALS) results. The red line indicates the experimental molecular weight. (c) The trimeric structure of the TRAF4 TRAF domain with a coiled-coil domain. A close-up of the interacting residues in the interface between two monomeric TRAF4 TRAF domains with a coiled-coil domain (blue for chain $A$ and yellow for chain $B$ ) is shown on the right. The residues involved in the contact are shown. The salt bridge formed between Glu291 from one chain and Gln293 from its counterpart is shown as a red dashed line. Hydrogen bonds are shown as black dashed lines.

\subsection{PDB accession code}

Coordinates and structural factors were deposited in the Protein Data Bank as PDB entry 4k8u.

\section{Results and discussion}

\subsection{Structure of the TRAF4 TRAF domain with a coiled-coil domain}

TRAF4 is composed of several distinct domains, including a RING domain, a zinc-finger domain, a coiled-coil domain and a TRAF domain (Fig. 1a). The TRAF domain is located at the C-terminus of TRAF4 and the coiled-coil domain is located immediately before the TRAF domain. The TRAF domain participates in protein-protein interactions in many important signalling pathways.
The $2.3 \AA$ resolution crystal structure of the TRAF domain with a coiled-coil domain comprising amino acids 290-470 was solved using the molecular-replacement (MR) method and was refined to an $R_{\text {work }}$ of $23.7 \%$ and an $R_{\text {free }}$ of $28.1 \%$. The high-resolution structure of the TRAF4 TRAF domain with a coiled-coil domain revealed that it is comprised of three helices, $\alpha 1-\alpha 3$, eight sheets, $\beta 1-\beta 8$, and an extra helix, $\alpha_{\mathrm{N}}$, at the N-terminus (Fig. $1 b$ ). The overall fold was similar to that of other TRAF domains. $\beta 2$, which was found in the structures of TRAF2 and TRAF6, was not detected in TRAF4, while $\alpha 3$, which is absent only in TRAF2, was detected in TRAF4. $\beta 7^{\prime}$, which is located before $\beta 7$, is only detected in TRAF4.

The structure of the TRAF4 TRAF domain is compact and ordered in the central region, with several disordered regions. The average $B$ factor is $69 \AA^{2}$ (Table 1). Plotting individual $B$ factors for each residue showed that the stacked $\beta$-sheet in 


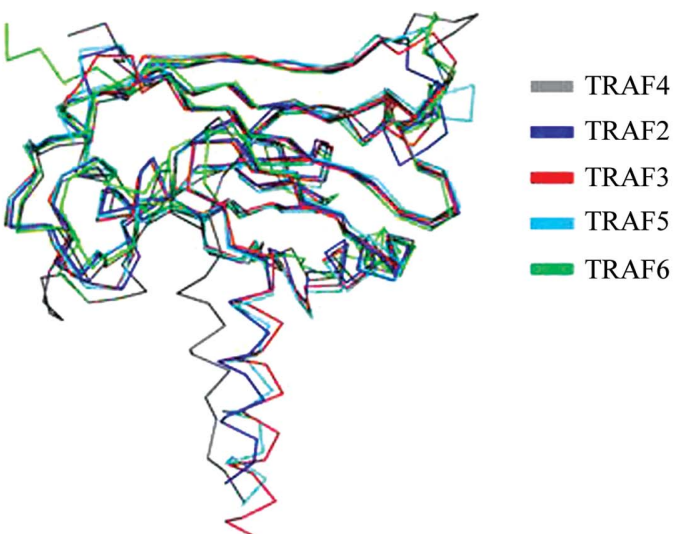

$(a)$

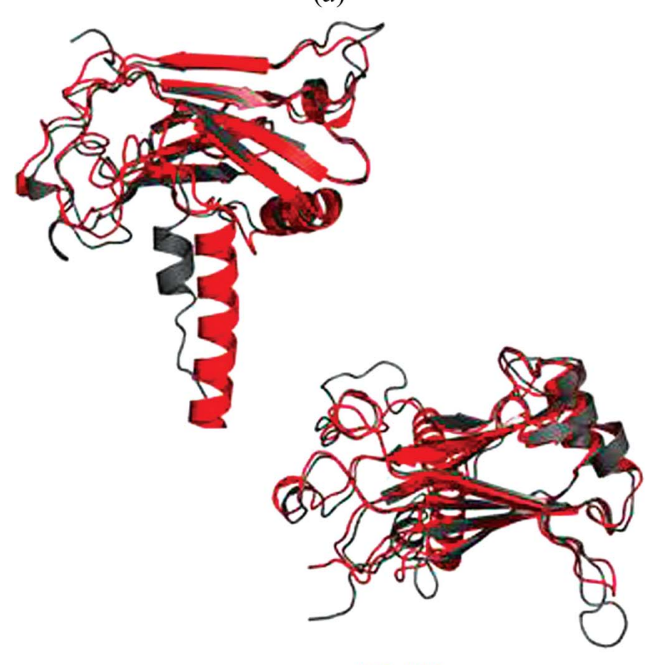

TRAF4 with TRAF3

(c)
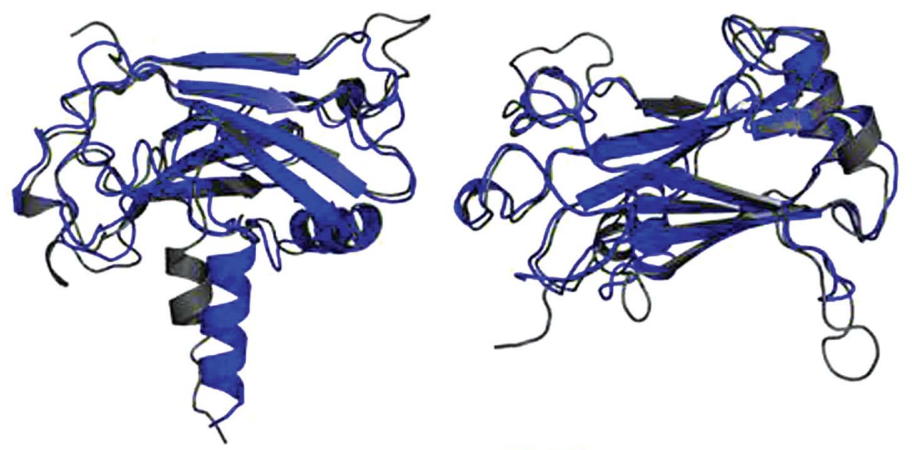

TRAF4 with TRAF2

(b)

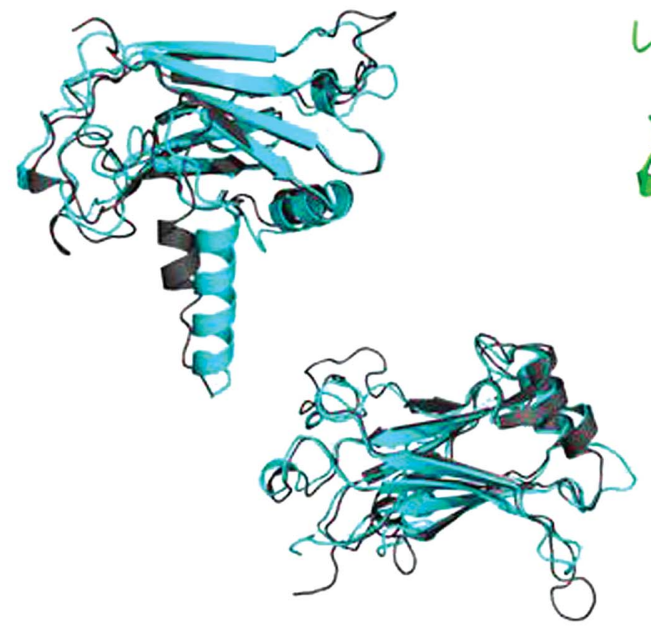

TRAF4 with TRAF5

(d)

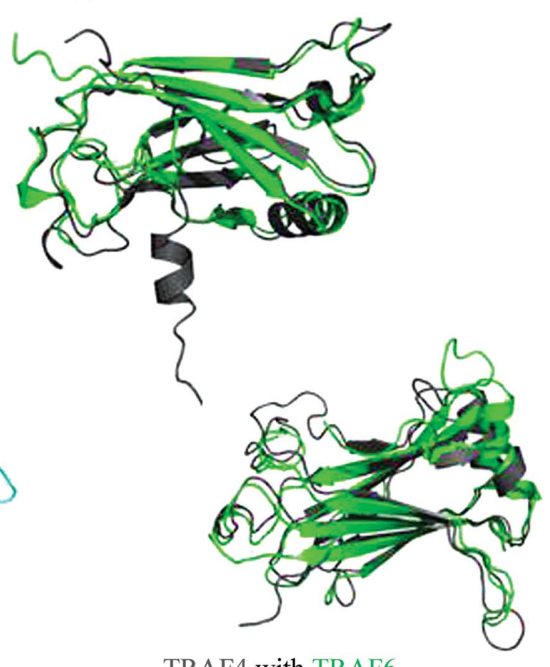

TRAF4 with TRAF6

(e)

Figure 3

Superposition of the TRAF4 TRAF domain with other TRAF family members. (a) The TRAF4 TRAF domain is superimposed with four structural homologues (grey, TRAF4; blue, TRAF2; red, TRAF3; cyan, TRAF5; green, TRAF6; blue, NALP1 PYD). Pairwise structural comparisons were also performed. TRAF4 is coloured grey and the counterparts are coloured blue for TRAF2 $(b)$, red for TRAF3 $(c)$, cyan for TRAF5 $(d)$ and green for TRAF6 (e). Side and top views are shown on the left and the right, respectively.

the central region has the lowest $B$ factors, whereas the long extended loop between $\beta 6$ and $\beta 7^{\prime}$ has the highest $B$ factors (Supplementary Fig. $\mathrm{S}^{\mathbf{1}}$ ). The $\mathrm{N}$ - and $\mathrm{C}$-terminal residues are also more flexible with higher $B$ factors.

There were three monomers in the asymmetric unit, chain $A$, chain $B$ and chain $C$ (Fig. 1c). A model of chain $A$ was built from residues 291 to 468 , while those of chain $B$ and chain $C$ were built from residues 293 to 468 and from residues 295 to 470 , respectively. Together, these chains formed a symmetric trimer. The seven antiparallel $\beta$-sheets comprising residues 309-314, 345-351, 362-370, 386-390, 404-408, 439-441 and $456-462$ were numbered $\beta 1, \beta 3, \beta 4, \beta 5, \beta 6$ and $\beta 7$, respectively (Fig. $1 b$ ). The probable $\beta$-sheets which were detected in TRAF2 and TRAF6, and comprised residues 331-337, are indicated as $(\beta 2)$. An extra sheet comprising residues 434-436 is indicated as $\left(\beta 7^{\prime}\right)$. Four $\alpha$-helices, $\alpha$ n (residues $\left.291-302\right), \alpha 1$

\footnotetext{
${ }^{1}$ Supporting information has been deposited in the IUCr electronic archive (Reference: MN5037).
}

(residues 316-327), $\alpha 2$ (residues 375-377) and $\alpha 3$ (residues 442-446), were detected (Fig. 1b).

\subsection{Stoichiometry of TRAF4 and the trimeric interface within the TRAF4 TRAF domain with a coiled-coil domain}

Based on previously reported TRAF structures, the TRAF domain was shown to exist as a trimer in solution (Park et al., 1999; Zhang et al., 2012; Ye et al., 2002). In the crystals of TRAF2 and TRAF6 the asymmetric unit contained a trimeric molecule, while the asymmetric unit of the TRAF2 and TRAF5 crystals contained a monomeric molecule (Park et al., 1999; Zhang et al., 2012; Ye et al., 2002).

The TRAF4 TRAF domain with a coiled-coil domain that was used in our structural study was overexpressed in bacteria and was purified by affinity chromatography followed by gelfiltration chromatography. On gel-filtration chromatography, TRAF4 eluted at around $15 \mathrm{ml}$, indicating that it forms a trimer in solution (Fig. 2a). The stoichiometry of TRAF4 was accurately confirmed by multi-angle light scattering (MALS). 
The calculated molecular weight of the monomeric TRAF4 TRAF domain with a coiled-coil domain (amino acids 290470) including the $\mathrm{C}$-terminal His tag was $21.9 \mathrm{kDa}$ and the experimental molecular weight from MALS was $71.2 \mathrm{kDa}$ ( $0.8 \%$ fitting error), with a polydispersity of 1.000 (Fig. $2 b$ ). According to the analysis using gel-filtration chromatography and MALS, we concluded that TRAF4 exists as a trimer in solution and that trimeric TRAF4, as detected in our crystal structure, might be a biologically functional unit.

The trimeric TRAF4 TRAF domain with a coiled-coil domain exhibited a typical mushroom shape, in which the TRAF domain forms the cap and the coiled-coil domain forms the stalk (Fig. $2 c$ ). The trimeric interface of TRAF4 is formed by packing one end of the ( $\beta 2)-\beta 3$ connecting loop, the $\beta 4-\beta 5$ connecting loop and $\alpha$ n of one monomer against an edge and a face of the $\beta 5-\beta 6$ connecting loop and $\beta 1$ of the neighbouring monomer (Fig. $2 c$ ). Several hydrogen bonds formed between Tyr342, Ala373, Asn376 and Trp380 from one TRAF molecule (chain $A$ ) and Val309, Ile311, Lys313, Gln393, Asp453 and Asp495 from the second molecule (chain $B$ ) and one main salt bridge between Glu291 in one monomer (chain $A$ ) and Gln293 in the neighbouring monomer (chain $B$ ) are key interactions for trimerization (Fig. 2c). The salt bridge in the trimeric interface was located in the coiled-coil stalk region, indicating that the coiled-coil domain is important for stabilization of trimer formation. The total surface area of the three molecules is around $27438 \AA^{2}$, and $3938 \AA^{2}$ is buried upon complex formation, which corresponds to an average of $1312 \AA^{2}$ per molecule. An average surface area of $656 \AA^{2}$ is buried upon dimer interface formation.

\subsection{Comparison with other TRAF-domain structures}

The $D A L I$ server was used to search for structurally similar proteins to the TRAF4 TRAF domain (Holm \& Sander, 1995) and several structurally related proteins were identified (Table 2). The top seven matches, which had $Z$-scores of
Table 2

Structure-similarity search using $D A L I$.

\begin{tabular}{|c|c|c|c|c|}
\hline Protein (PDB code) & $Z$-score & $\begin{array}{l}\text { R.m.s.d. } \\
(\AA)\end{array}$ & $\begin{array}{l}\text { Identity } \\
(\%)\end{array}$ & Reference \\
\hline TRAF2 (1czy) & 22.8 & 1.9 & 46 & Park et al. (1999) \\
\hline TRAF3 (4ghu) & 22.7 & 2.1 & 39 & Zhang et al. (2012) \\
\hline TRAF5 (4gjh) & 22.5 & 2.1 & 44 & Zhang et al. (2012) \\
\hline TRAF6 (1lb4) & 19.1 & 1.8 & 33 & Ye et al. (2002) \\
\hline Meprin (4gwn) & 15.8 & 2.7 & 27 & Arolas et al. (2012) \\
\hline $\begin{array}{l}\text { Speckle-type } \mathrm{POZ} \text { protein } \\
(3 \mathrm{hqm})\end{array}$ & 13.7 & 2.4 & 17 & Zhuang et al. (2009) \\
\hline HAUSP/USP7 (2f1y) & 12.7 & 3.0 & 10 & Hu et al. (2006) \\
\hline
\end{tabular}

22.8-12.7, were (in order) TRAF2, TRAF3, TRAF5, TRAF6, meprin, speckle-type POZ protein (SPOP) and HAUSP.

Pairwise structural alignments between the TRAF4 TRAF domain and other TRAF domains showed that the positions and the lengths of several loops in the TRAF4 TRAF domain differed from those in other TRAF domains (Fig. 3). In particular, two loops connecting $\beta 5-\beta 6$ and $\beta 6-\beta 7$ of the TRAF4 TRAF domain were relatively longer than those in other TRAFs (Fig. 3). The location of the $\alpha$ n helix of the coiled-coil domain also differed in that it did not superimpose well on the $\alpha$ helix in other TRAFs. These structural differences of TRAF4 might be critical to its functional differences from other TRAFs. The trimeric structure of the TRAF4 TRAF domain was also superimposed with trimeric TRAF2 and TRAF5 (Fig. 4). TRAF4 and TRAF2 superimposed with a root-mean-square deviation (r.m.s.d.) of $2.2 \AA$, while TRAF4 and TRAF5 superimposed with an r.m.s.d. of $2.4 \AA$. This structural similarity indicated that the TRAF domains shared the common feature of trimer formation.

Because the TRAF domain of TRAFs interacts with diverse receptors including TNF-R2, CD40 and RANK, the structures of several complex structures between TRAF and its receptors have been solved. The complex structures indicate that $\beta 3$, $\beta 4, \beta 6$ and $\beta 7$ of the TRAF domains participate in receptor interactions (Fig. 5a). Since the surface features often dictate the mode of interaction with partners (Bae \& Park, 2011; Park,

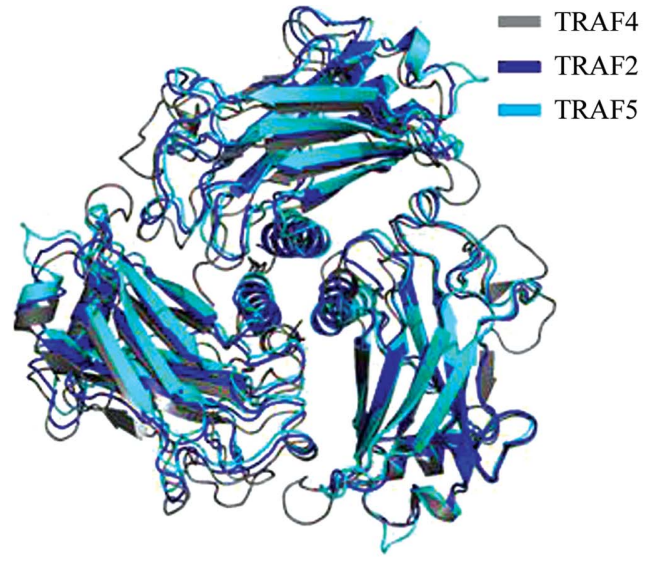

(a)

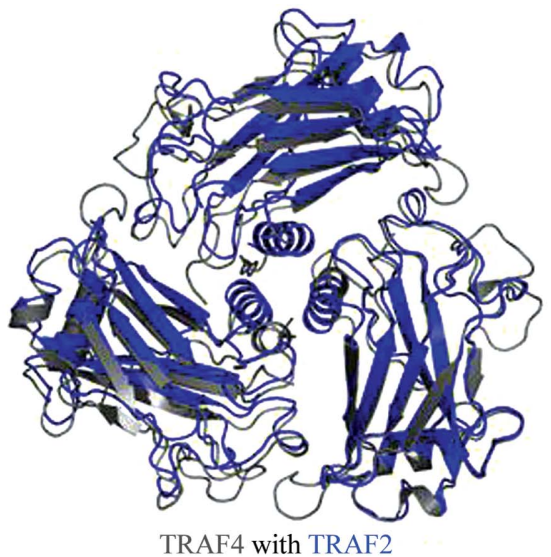

(b)

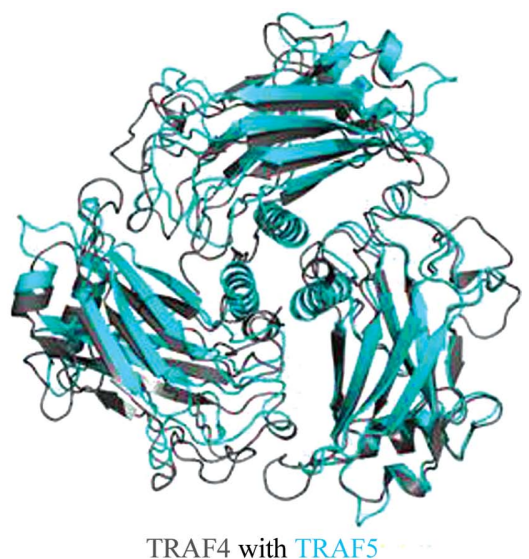

(c)

Figure 4

Superposition of the trimeric TRAF4 TRAF domain with trimeric TRAF2 and TRAF5. (a) TRAF4 TRAF domain superimposed with two trimeric TRAF domains (grey, TRAF4; blue, TRAF2; cyan, TRAF5). A pairwise structural comparison was also performed. TRAF4 is coloured grey and the counterparts are coloured blue for TRAF2 $(b)$ and cyan for TRAF5 $(c)$. 
2011), the electrostatic surface of the TRAF4 TRAF domain was calculated and compared with those of other TRAFs. The TRAF domain of TRAF4 has similar gross features on its electrostatic surface as the other TRAFs (Figs. 5b-5f). For example, the charge nature of most other TRAFs (with the exception of the surface of TRAF6, which contains primarily positive charges) is mixed, as is that of the TRAF4 TRAF domain surface. Because the diversely charged surface of the TRAFs has been shown to be important for accommodating many diverse receptors in the same binding pocket, TRAF4 might also be involved in many signalling pathways via interactions with diverse receptor families.

\subsection{Mapping of conserved residues that might be critical for protein interactions}

To identify the residues that might be involved in protein interactions for signalling events, the TRAF4 sequence was aligned with those of other TRAF family members (TRAF2, TRAF3 and TRAF6) that had been elucidated and mapped in previous studies (Ely \& Li, 2002; Ye et al., 2002; Ye, Park et al., 1999; Ni et al., 2000). Structural information describing the complexes of TRAFs with various receptors, including CD40, TRADD, LMP1, TNFR2, RANK and TANK, is available (Ye, Park et al., 1999; Ye et al., 2002; Park et al., 1999, 2000). Surface residues that have been shown to participate in receptor interactions with TRAF2, TRAF3 and TRAF6 were marked on the sequences (Fig. 6a). A structure-based sequencealignment and interaction-mapping study showed that the binding receptors of the TRAF family interact using three regions: (i) $\beta 3$ and the loop connecting $\beta 3$ and $\beta 4$, (ii) $\beta 6$ and the loop connecting $\beta 6$ and $\beta 7$ and (iii) $\beta 7$. Among those residues involved in the three interaction regions, conserved residues which might also be involved in the interaction of TRAF4 with its receptor were marked on the sequence of TRAF4 and mapped onto the surface of the TRAF4 TRAF domain (Fig. 6b). A number of residues on the surface that are involved in receptor interaction are conserved among TRAF domains (Figs. $6 a$ and 6b). These include Ser351, Gly356, Ser357, Tyr366, Phe408, Pro410, Asp411, Leu432, Gly433, Phe434 and Tyr436. Because the TRAF family uses the same binding sites (previously called hot spots 1,2 and 3) composed of various amino-acid residues to accommodate various different receptors, we considered residues in TRAF4 that are

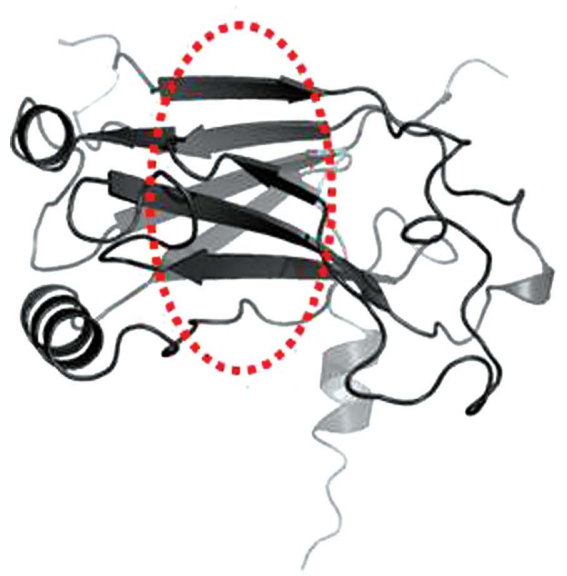

(a)

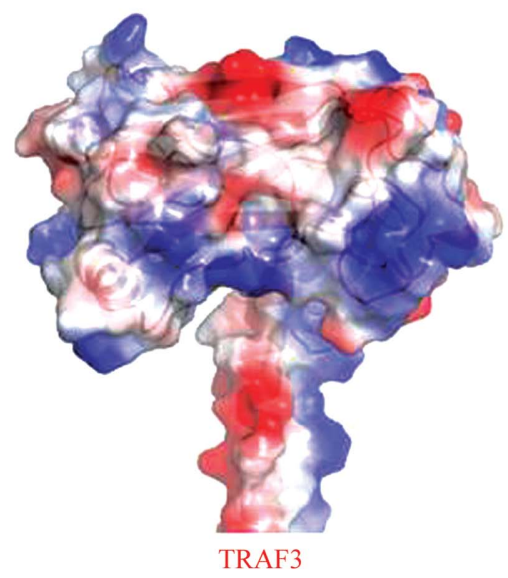

(d)

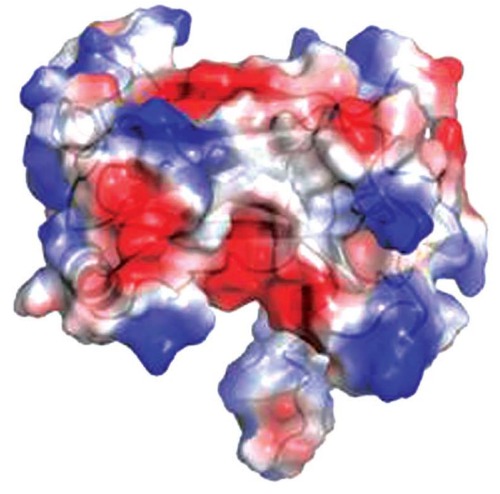

TRAF4

(b)

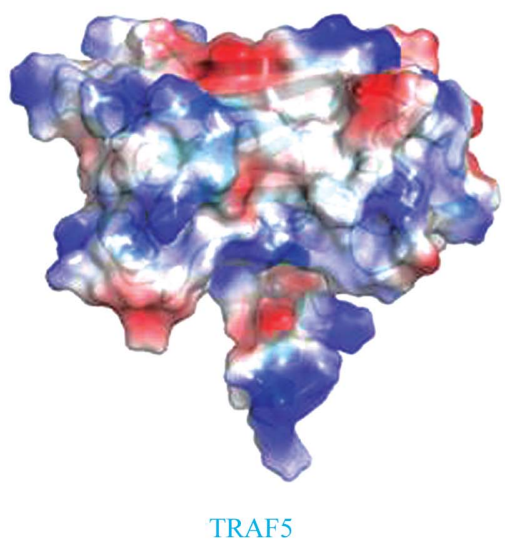

(e)

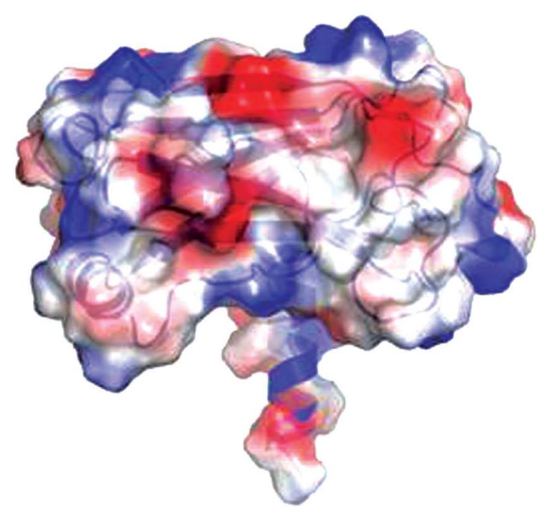

TRAF2

(c)

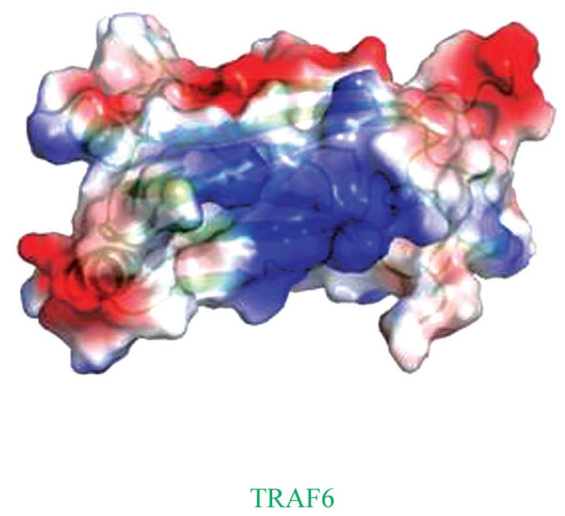

(f)

Figure 5

Electrostatic surface representations of the TRAF4 TRAF domain and its structural homologues. (a) Orientation of the TRAF domain for surface generation. The receptor-binding pocket of the TRAF family is indicated by the red dotted circle. $(b)-(f)$ Electrostatic surface representations of TRAF domains generated by Coot. 


\section{TRAF4 TRAF2 TRAF3 TRAF 6 \\ TRAF 4 TRAF2 TRAF3 TRAF 6}

321 LQEAKAKPNLECFSPAFYTHKYGY Ǩ…................. SÄGET-HLSLYIRVLPGAFDNLLE 379 365 RQEAVAGRIPAIFSPAFYTSRYGYKMCLRIYLNGDGT'̈RGT-HLSLFFVVMKGPNDALLR 423 429 KQEAVMGKTLSLYSQP FYTGYFGYKMCARVYLNGDGM:KGT-HLSLFFVIMRGEYDALLP 487 364 LKCQEEEKPVVIHSPGFYTGKPGYKLCMRLHLQLPTAQRCANYISLFVHTMQGEYDSHLP 423

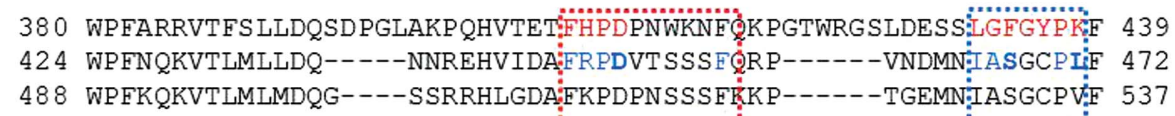
424 WPEQGTIRLT ILDQSE--APVRQNHEEIMDAKPELIAFQRP-----TIPRNP'KGFGYVTF 476

(a)

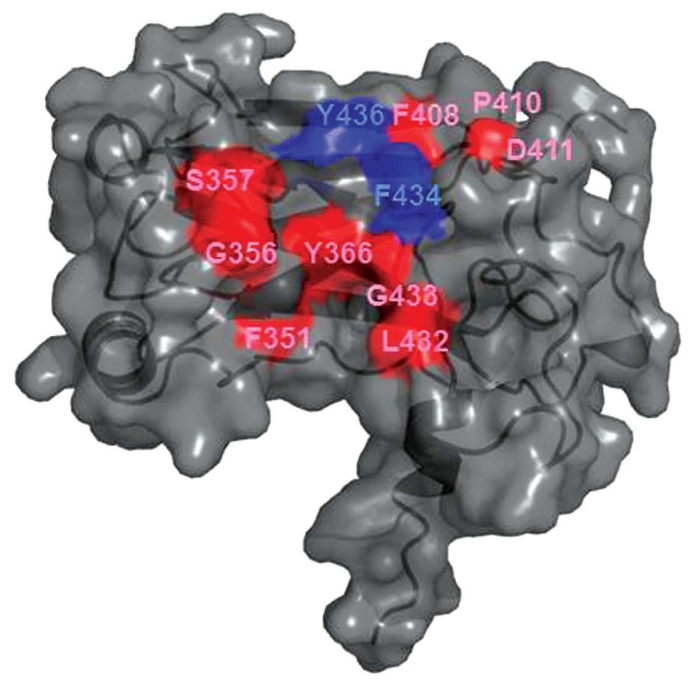

(b)

\section{Figure 6}

Mapping of conserved exposed residues onto the TRAF4 TRAF domain with a coiled-coil domain. (a) Structure-based sequence alignment. The surface residues involved in interaction with binding receptors in TRAF2, TRAF3 and TRAF6 are shown in blue. Conserved exposed residues in TRAF4 are shown in red. The three interaction regions in the TRAF domain are indicated by a black dotted square (first region), a red dotted square (second region) and a blue dotted circle (third region). (b) Conserved exposed residues that might be involved in receptor interaction are mapped onto the TRAF4 TRAF domain. Red residues are deduced from structural information on the complex between TRAF2 or TRAF3 and their binding receptors. Blue residues are deduced from structural information on the complex between TRAF6 and its binding receptors.

conserved in one or more TRAFs as conserved residues that might be important for receptor interaction. The mapping analysis suggests that this extensive surface of the TRAF4 TRAF domain, which contains both hydrophobic and charged residues, may be involved in interactions among various receptors. Further structural studies, especially of the complex between TRAF4 and its binding receptor, are needed to elucidate the TRAF4-mediated signalling event. In conclusion, although the signalling events following receptor interactions are vague, the structure of the TRAF4 TRAF domain with a coiled-coil domain presented here provides a first step towards elucidation of the molecular basis of the TRAF4 signalling pathway.

This study was supported by the Basic Science Research Program through the National Research Foundation of Korea (NRF) of the Ministry of Education, Science and Technology (2013009083).

\section{References}

Arch, R. H., Gedrich, R. W. \& Thompson, C. B. (1998). Genes Dev. 12, 2821-2830.
Arolas, J. L., Broder, C., Jefferson, T., Guevara, T., Sterchi, E. E., Bode, W., Stöcker, W., Becker-Pauly, C. \& Gomis-Rüth, F. X. (2012). Proc. Natl Acad. Sci. USA, 109, 1613116136.

Aston, C., Jiang, L. \& Sokolov, B. P. (2004). J. Neurosci. Res. 77, 858-866.

Bae, J. Y. \& Park, H. H. (2011). Acta Cryst. F67, 1421-1424.

Bishop, G. A. (2004). Nature Rev. Immunol. 4, 775-786.

Bradley, J. R. \& Pober, J. S. (2001). Oncogene, 20, 6482-6491.

Chung, J. Y., Park, Y. C., Ye, H. \& Wu, H. (2002). J. Cell Sci. 115, 679-688.

Darnay, B. G., Ni, J., Moore, P. A. \& Aggarwal, B. B. (1999). J. Biol. Chem. 274, 7724-7731.

DeLano, W. L. \& Lam, J. W. (2005). Abstr. Pap. Am. Chem. Soc. 230, U1371-U1372.

Ely, K. R. \& Li, C. (2002). J. Mol. Recognit. 15, 286-290.

Emsley, P. \& Cowtan, K. (2004). Acta Cryst. D60, 2126-2132.

Hoeflich, K. P., Yeh, W.-C., Yao, Z., Mak, T. W. \& Woodgett, J. R. (1999). Oncogene, 18, 5814-5820.

Holm, L. \& Sander, C. (1995). Trends Biochem. Sci. 20, 478-480.

Hsu, H., Huang, J., Shu, H.-B., Baichwal, V. \& Goeddel, D. V. (1996). Immunity, 4, 387-396.

Hu, M., Gu, L., Li, M., Jeffrey, P. D., Gu, W. \& Shi, Y. (2006). PLoS Biol. 4, e27.

Inoue, J., Ishida, T., Tsukamoto, N., Kobayashi, N., Naito, A., Azuma, S. \& Yamamoto, T. (2000). Exp. Cell Res. 254, 14-24.

Izban, K. F., Ergin, M., Martinez, R. L. \& Alkan, S. (2000). Mod. Pathol. 13, 1324-1331.

Kedinger, V. \& Rio, M.-C. (2007). Adv. Exp. Med. Biol. 597, 60-71.

Laskowski, R. A., MacArthur, M. W., Moss, D. S. \& Thornton, J. M. (1993). J. Appl. Cryst. 26, 283-291.

Marinis, J. M., Homer, C. R., McDonald, C. \& Abbott, D. W. (2011). J. Biol. Chem. 286, 1938-1950.

McCoy, A. J. (2007). Acta Cryst. D63, 32-41.

Murshudov, G. N., Skubák, P., Lebedev, A. A., Pannu, N. S., Steiner, R. A., Nicholls, R. A., Winn, M. D., Long, F. \& Vagin, A. A. (2011). Acta Cryst. D67, 355-367.

Ni, C.-Z., Welsh, K., Leo, E., Chiou, C.-K., Wu, H., Reed, J. C. \& Ely, K. R. (2000). Proc. Natl Acad. Sci. USA, 97, 10395-10399.

Otwinowski, Z. \& Minor, W. (1997). Methods Enzymol. 276, 307-326.

Park, H. H. (2011). Apoptosis, 16, 209-220.

Park, Y. C., Burkitt, V., Villa, A. R., Tong, L. \& Wu, H. (1999). Nature (London), 398, 533-538.

Park, Y. C., Ye, H., Hsia, C., Segal, D., Rich, R. L., Liou, H.-C., Myszka, D. G. \& Wu, H. (2000). Cell, 101, 777-787.

Perrakis, A., Harkiolaki, M., Wilson, K. S. \& Lamzin, V. S. (2001). Acta Cryst. D57, 1445-1450.

Régnier, C. H., Masson, R., Kedinger, V., Textoris, J., Stoll, I., Chenard, M.-P., Dierich, A., Tomasetto, C. \& Rio, M.-C. (2002). Proc. Natl Acad. Sci. USA, 99, 5585-5590.

Rothe, M., Sarma, V., Dixit, V. M. \& Goeddel, D. V. (1995). Science, 269, 1424-1427. 
Rothe, M., Wong, S. C., Henzel, W. J. \& Goeddel, D. V. (1994). Cell, 78, 681-692.

Shiels, H., Li, X., Schumacker, P. T., Maltepe, E., Padrid, P. A., Sperling, A., Thompson, C. B. \& Lindsten, T. (2000). Am. J. Pathol. 157, 679-688.

Song, H. Y., Régnier, C. H., Kirschning, C. J., Goeddel, D. V. \& Rothe, M. (1997). Proc. Natl Acad. Sci. USA, 94, 9792-9796.

Song, H. Y., Rothe, M. \& Goeddel, D. V. (1996). Proc. Natl Acad. Sci. USA, 93, 6721-6725.

Xie, P. (2013). J. Mol. Signal. 8, 7.

Ye, H. et al. (2002). Nature (London), 418, 443-447.

Ye, X., Mehlen, P., Rabizadeh, S., VanArsdale, T., Zhang, H., Shin, H., Wang, J. J. L., Leo, E., Zapata, J., Hauser, C. A., Reed, J. C. \& Bredesen, D. E. (1999). J. Biol. Chem. 274, 3020230208.
Ye, H., Park, Y. C., Kreishman, M., Kieff, E. \& Wu, H. (1999). Mol. Cell, 4, 321-330.

Yoon, J. H. \& Park, H. H. (2013). Acta Cryst. F69, 1026-1028.

Zapata, J. M., Lefebvre, S. \& Reed, J. C. (2007). Adv. Exp. Med. Biol. 597, 188-201.

Zapata, J. M., Pawlowski, K., Haas, E., Ware, C. F., Godzik, A. \& Reed, J. C. (2001). J. Biol. Chem. 276, 24242-24252.

Zepp, J. A., Liu, C., Qian, W., Wu, L., Gulen, M. F., Kang, Z. \& Li, X. (2012). J. Immunol. 189, 33-37.

Zhang, P., Reichardt, A., Liang, H. H., Aliyari, R., Cheng, D., Wang, Y. Y., Xu, F., Cheng, G. H. \& Liu, Y. F. (2012). Sci. Signal. 5, ra81. Zhuang, M., Calabrese, M. F., Liu, J., Waddell, M. B., Nourse, A., Hammel, M., Miller, D. J., Walden, H., Duda, D. M., Seyedin, S. N., Hoggard, T., Harper, J. W., White, K. P. \& Schulman, B. A. (2009). Mol. Cell, 36, 39-50. 